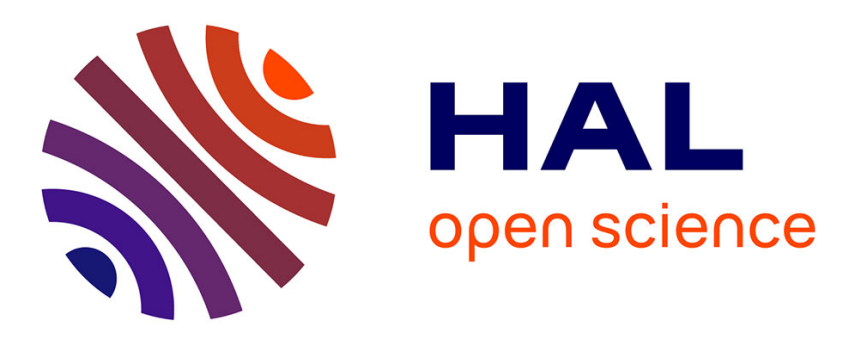

\title{
Diffusion in bulk liquids: finite-size effects in anisotropic systems
}

Alexandru Botan, Virginie Marry, Benjamin Rotenberg

\section{To cite this version:}

Alexandru Botan, Virginie Marry, Benjamin Rotenberg. Diffusion in bulk liquids: finitesize effects in anisotropic systems. Molecular Physics, 2015, 113 (17-18), pp.2674-2679. 10.1080/00268976.2015.1021730 . hal-01484356

\section{HAL Id: hal-01484356 https: / hal.sorbonne-universite.fr/hal-01484356}

Submitted on 8 Nov 2018

HAL is a multi-disciplinary open access archive for the deposit and dissemination of scientific research documents, whether they are published or not. The documents may come from teaching and research institutions in France or abroad, or from public or private research centers.
L'archive ouverte pluridisciplinaire HAL, est destinée au dépôt et à la diffusion de documents scientifiques de niveau recherche, publiés ou non, émanant des établissements d'enseignement et de recherche français ou étrangers, des laboratoires publics ou privés. 


\title{
Diffusion in bulk liquids: finite-size effects in anisotropic systems
}

\author{
Alexandru Botan ${ }^{a}$, Virginie Marry $^{b, c}$, Benjamin Rotenberg $^{b, c *}$ \\ ${ }^{a}$ Centre for Materials Science and Nanotechnology \& Department of Physics, University \\ of Oslo, 0316 Oslo, Norway; \\ ${ }^{b}$ Sorbonne Universités, UPMC Univ Paris 06, UMR PHENIX, F-75005, Paris, France; \\ ${ }^{c}$ CNRS, UMR PHENIX, F-75005, Paris, France; \\ (January 16, 2015)
}

\begin{abstract}
We investigate systematically the effect of the cell size and shape on the diffusion properties in MD simulations. Specifically, we consider a bulk LJ fluid in orthorhombic cells with one length differing from the other two. The components of the diffusion tensor display complex variations as a function of the two independent lengths and may even become in some cases larger than the macroscopic limit for a cubic cell. These results can be perfectly explained by a purely hydrodynamic theory, which extends results obtained previously for the isotropic case. We provide the explicit expression of the diffusion tensor, including the effect of the finite size of the diffusing particle. The simulation results follow a simple scaling as a function of box size and aspect ratio and the corresponding scaling functions are determined numerically. These findings should have implications for the practically more relevant case of confined fluids.
\end{abstract}

\section{Introduction}

The diffusion of a solute or a solvent molecule arises from momentum transfer with the surrounding fluid, a process also at the origin of hydrodynamic flows around the particle - hence of hydrodynamic interactions between solutes. This fundamental correspondence is evident in the Stokes-Einstein relation, $D_{\infty}=k_{B} T / 6 \pi \eta a$, relating the diffusion coefficient of a spherical particle of radius $a$ to the solvent viscosity $\eta$ (and the temperature $T$, with $k_{B}$ Boltzmann's constant), which can be derived from macroscopic hydrodynamics assuming stick boundary conditions at the surface.

One consequence of practical importance is that the computation of diffusion coefficients with Molecular Dynamics (MD) simulation suffers from finite-size effects arising from hydrodynamic interactions between periodic images $[1,2]$. For a cubic simulation box, the overall effect for a bulk fluid is a decrease in the diffusion coefficient. This decrease numerically follows down to almost molecular sizes the following scaling:

$$
D(L)=D_{\infty}-\frac{c k_{B} T}{6 \pi \eta L}
$$

with $L$ the box length and $c \approx 2.837$. The derivation of this result, already reported by Dünweg and Kremer [2] and by Yeh and Hummer [1] uses the Oseen tensor to

*Corresponding author. Email: benjamin.rotenberg@upmc.fr 
compute the flow generated by the particle under a force $\mathbf{F}$ and Ewald summation to account for periodicity. They found that most of this effect is in fact due to the enforcement of a background force density $-\mathbf{F} / V$, with $V$ the volume of the system, ensuring overall momentum conservation. Higher order corrections accounting for the finite size of the solute can be obtained by considering the Rotne-Prager instead of the Oseen tensor $[1,3,4]$.

Most reported simulation data, even since the publication of these results, neglect this contribution and therefore underestimate the diffusion coefficient. Nevertheless, this scaling law has been verified numerically for a number of systems, including several popular water models [5-7], ionic liquids [8] or more complex fluids such as solutions of star polymers [9] and even in higher dimensional spaces [10]. This scaling then allows extrapolating the solvent diffusion coefficient to the macroscopic $L \rightarrow \infty$ limit, or the correction to be applied for solutes $[11,12]$. In addition, the slope provides an estimate of the viscosity - a strategy that was used in particular to determine the viscosity of water in ab initio MD simulations [13].

More recently, it was observed that for anistropic simulation boxes, the diffusion tensor becomes anisotropic even for bulk fluids [6]. Such finite-size artefacts may become problematic for the study of confined fluids, a very active field of research due to the numerous applications in a wide variety of contexts (e.g. micro- and nanofluidics, nanoporous materials, etc), for which anisotropic simulation boxes are the rule rather than the exception. Note that in the case of a slit pore, one dimension is indeed finite and only the periodicity in the other directions results in spurious size effects. For an anisotropic bulk water system, Rozmanov and Kusalik found that replacing $L$ by $V^{-1 / 3}$ in Eq. 1 is not sufficient to describe the components of the diffusion tensor. Rather, they proposed for the considered geometry to use instead the square root of the cross-sectional area corresponding to each direction [6].

Here we investigate systematically the effect of simulation box anisotropy on the diffusion tensor. We show that hydrodynamic effects can explain the simulation results, including the fact that some components may exceed the extrapolated value for a cubic box in the infinite size limit. To that end, we follow the strategy of Ref. [1] and consider explicitely (a) the Rotne-Prager tensor instead of Oseen, to account for the finite size of the diffusion particle (b) all components of the diffusion tensor instead of the isotropic part. This is presented in Section 2, together with the simulation methods. Section 3 briefly reports the results for isotropic boxes. The main results of the present work, dealing with anisotropic boxes, are finally presented in Section 4.

\section{Hydrodynamic theory and simulation methods}

\subsection{Hydrodynamics}

The effect of hydrodynamics on the diffusion tensor in periodic systems, as well as explicit expressions for its isotropic component and for cubic boxes has been given by Yeh and Hummer [1]. For the sake of completeness, we first recall the main arguments of their derivation and then extend it to provide the expressions for the full tensor, including the effect of the finite size of the particle via the Rotne-Prager tensor. The diffusion tensor under periodic boundary conditions is given by:

$$
\mathbb{D}_{\mathrm{PBC}}^{a}=D_{\infty} \mathbb{1}+k_{B} T \lim _{r \rightarrow 0}\left[\mathbb{T}_{\mathrm{PBC}}(\mathbf{r})-\mathbb{T}_{\infty}(\mathbf{r})\right],
$$

where $\mathbb{T}_{\text {PBC }}$ and $\mathbb{T}_{\infty}$ denote the mobility tensors under periodic and unbounded conditions, respectively, and $\mathbb{1}$ is the identity matrix. These tensors relate the 
velocity of the fluid at a position $\mathbf{r}$ around the particle when the latter is submitted to an applied force $\mathbf{F}$, while a compensating background force $-\mathbf{F} / V$ is distributed uniformly over the fluid, as $\mathbf{v}(\mathbf{r})=\mathbb{T}(\mathbf{r}) \cdot \mathbf{F}$. For point particles, the mobility tensor in the unbounded case is the Oseen tensor:

$$
\mathbb{T}_{\infty}^{0}(\mathbf{r})=\frac{1}{8 \pi \eta r}\left(\mathbb{1}+\frac{\mathbf{r r}}{r^{2}}\right)
$$

where the superscript refers to the vanishing radius $a=0$, while for the periodic case it is more conveniently written in reciprocal space as:

$$
\mathbb{T}_{\mathrm{PBC}}^{0}(\mathbf{r})=\sum_{\mathbf{k} \neq 0} \frac{e^{-i \mathbf{k} \cdot \mathbf{r}}}{\eta k^{2} V}\left(\mathbb{1}-\frac{\mathbf{k k}}{k^{2}}\right)
$$

where the sum extends over the reciprocal lattice vectors $\mathbf{k}$, excluding $k=0$. For spheres of radius $a \neq 0$, the Oseen tensor is replaced by the Rotne-Prager tensor [3] and the extension of Eq. 4 has been given by Hasimoto [4] and Beenakker [14]. The diffusion tensor can then be written as an Ewald sum by introducing a real number $\xi>0$ :

$$
\mathbb{D}_{\mathrm{PBC}}=D_{\infty} \mathbb{1}+\frac{k_{B} T \xi}{6 \pi \eta}\left[\left(-6+\frac{40 a^{2} \xi^{2}}{3}\right) \frac{1}{\sqrt{\pi}} \mathbb{1}+\mathbb{M}_{\mathbf{r}}(a)+\frac{6 \pi}{V \xi^{3}} \mathbb{M}_{\mathbf{k}}(a)\right]
$$

where we have introduced two tensors:

$$
\begin{aligned}
\mathbb{M}_{\mathbf{r}}(a) & =\sum_{\mathbf{s} \neq 0}\left\{f_{1}(s) \mathbb{1}+f_{2}(s) \frac{\mathbf{s s}}{s^{2}}\right\} \\
f_{1}(s) & =\left(\frac{3}{4}+\frac{a^{2} \xi^{2}}{2 s^{2}}\right) \frac{\operatorname{erfc} s}{s}+\left(-\frac{9}{2}+14 a^{2} \xi^{2}+3 s^{2}-20 a^{2} \xi^{2} s^{2}+4 a^{2} \xi^{2} s^{4}+\frac{a^{2} \xi^{2}}{s^{2}}\right) \frac{e^{-s^{2}}}{\sqrt{\pi}} \\
f_{2}(s) & =\left(\frac{3}{4}-\frac{3 a^{2} \xi^{2}}{2 s^{2}}\right) \frac{\operatorname{erfc} s}{s}+\left(\frac{3}{2}-2 a^{2} \xi^{2}-3 s^{2}+16 a^{2} \xi^{2} s^{2}-4 a^{2} \xi^{2} s^{4}-\frac{3 a^{2} \xi^{2}}{s^{2}}\right) \frac{e^{-s^{2}}}{\sqrt{\pi}}
\end{aligned}
$$

and

$$
\mathbb{M}_{\mathbf{k}}(a)=\sum_{\mathbf{t} \neq 0} \frac{e^{-t^{2} / 4}}{t^{2}}\left\{1+\frac{t^{2}}{4}+\frac{t^{4}}{8}\right\}\left\{1-\frac{a^{2} \xi^{2} t^{2}}{3}\right\}\left(\mathbb{1}-\frac{\mathbf{t t}}{t^{2}}\right)
$$

The two sums extend over real and reciprocal space lattice vectors, respectively. For an orthorhombic box with lengths $L_{x}, L_{y}$ and $L_{z}$, to which we will restrict ourselves in the following, this reads:

$$
\begin{aligned}
& \mathbf{s}=n_{x} \xi L_{x} \mathbf{e}_{x}+n_{y} \xi L_{y} \mathbf{e}_{y}+n_{z} \xi L_{z} \mathbf{e}_{z} \\
& \mathbf{t}=p_{x} \frac{2 \pi}{\xi L_{x}} \mathbf{e}_{x}+p_{y} \frac{2 \pi}{\xi L_{y}} \mathbf{e}_{y}+p_{z} \frac{2 \pi}{\xi L_{z}} \mathbf{e}_{z}
\end{aligned}
$$

with $n_{x, y, z}$ and $p_{x, y, z}$ integers and where $s=\|\mathbf{s}\|$ and $t=\| \mathbf{t}||$ denote the norm of these vectors.

Eqs. 5-9 provide the extension of Eq. 1 to finite size solutes and to anisotropic simulation boxes, where the components of the diffusion tensor may differ. Eq. 1 is recovered in the $a \rightarrow 0$ and taking the isotropic part $\left(\frac{1}{3} \operatorname{Tr} \mathbb{D}_{\mathrm{PBC}}\right)$. In the more 
general case we were not able to compute the Ewald sums analytically, however they can be easily determined numerically provided a suitable choice of $\xi$ and number of terms in the sums 6 and 7 . In the following, results are obtained by choosing $\xi=6.4 / \min _{\alpha} L_{\alpha}, n_{x, y, z} \in\left[-n_{\max }, n_{\max }\right]$ with $n_{\max }=1$ and $p_{x, y, z} \in$ $\left[-p_{\max }, p_{\max }\right]$ with $p_{\max }=2 \xi\left\lfloor\min _{\alpha} L_{\alpha}\right\rfloor+1$, which are sufficient to ensure good convergence. For a systematic study of the effect of anisotropy, we consider for simplicity orthorhombic systems where two lengths are identical. Without loss of generality, we note $L_{x}=L_{y}=L_{\|}$and $L_{z}=L_{\perp}$ (see Figure 1). In that case, the diffusion tensor is diagonal with $D_{x x}=D_{y y}=D_{\|}$and $D_{z z}=D_{\perp}$.

\subsection{Simulation details}

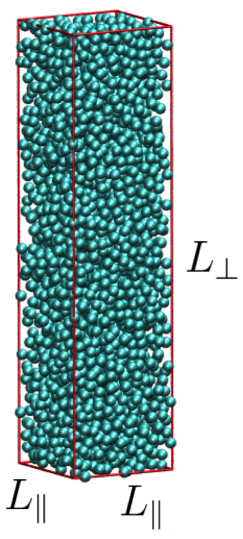

Figure 1. The simulated systems consist of a LJ fluid in orthorhombic cells with one length different from the other two. Both elongated $\left(L_{\perp}>L_{\|}\right.$, as shown) and flat $\left(L_{\perp}<L_{\|}\right)$are considered.

We simulate a Lennard-Jones fluid under the same physical conditions as the one considered in Ref. [1], namely a reduced density $\rho^{*}=\rho \sigma^{3}=0.7$ and a reduced temperature $T^{*}=k_{B} T / \epsilon=2.75$, with $\sigma$ and $\epsilon$ the Lennard-Jones diameter and energy, in the supercritical fluid region. The simulated systems, illustrated in Figure 1, cover a range of sizes and aspect ratios from flat to elongated. Specifically, the box lengths corresponding to the results reported below are summarized in table 1, with particle numbers ranging from 87 to 87500 .

\begin{tabular}{l|c|c|}
\hline$L_{\|}$ & $L_{\perp}$ \\
\hline $5,10,15,20,30,40,50$ \\
\hline 10 & $5,10,20,40,80$ \\
40 & $5,10,20,40$ \\
\hline $5,10,20,40,80$ & 10 \\
$5,10,20,40$ & 40 \\
\hline
\end{tabular} to cubic cells.

Molecular simulations are performed in the microcanonical ensemble for $10^{4} t^{*}$, with $t^{*}=\sigma \sqrt{m / \epsilon}$ the Lennard-Jones time unit, using a time step of $10^{-3} t^{*}$, after an equlibration period of $10^{2} t^{*}$ in the canonical ensemble. For the latter, a NoseHoover thermostat with a time constant of $0.1 t^{*}$ is used. A cut-off of $2.5 \sigma$ is used to compute the Lennard-Jones interactions. For each system, 8 independent runs (16 for the smallest system $L_{\|}=L_{\perp}=5$ ) with different initial conditions are performed. For each simulation, the mean-square displacement in each direction 
is computed and the components of the diffusion tensor are determined from the slope for $t<4.10^{3} t^{*}$. Reported results are averages over these independent runs, with errorbars computed using the block averaging method [15].

Since the hydrodynamic results involve the viscosity of the fluid, we also compute this quantity from equilibrium simulations using the Green-Kubo expression:

$$
\eta=\frac{V}{k_{B} T} \int_{0}^{\infty}\left\langle\sigma_{\alpha \beta}(t) \sigma_{\alpha \beta}(0)\right\rangle \mathrm{d} t
$$

involving the auto-correlation function $(\mathrm{ACF})$ of the off-diagonal components of the stress tensor $\sigma_{\alpha \beta}$. The reported results for the viscosity correspond to the $L_{\|}=L_{\perp}=15$ system. As previously noted in the literature [1], we did not observe significant size effect on the viscosity. All simulations are performed using the LAMMPS simulation package [16].

\section{Isotropic boxes}

Results for isotropic boxes $\left(L_{\|}=L_{\perp}=L\right)$ are reported in Figure 2. As expected, they follow the linear dependence in $1 / L$ of Eq. 1). The value of the viscosity, $\eta=1.28 \pm 0.03$, is in good agreement with Ref. [1] and describes very well the slope of $D$ vs $1 / L$. The extrapolated value for infinite box size, $D_{\infty}=0.312 \pm 0.005$ is also consistent with Ref. [1]. These values of $\eta$ and $D_{\infty}$ are then used in the following for the hydrodynamic calculations in the anistropic cases.

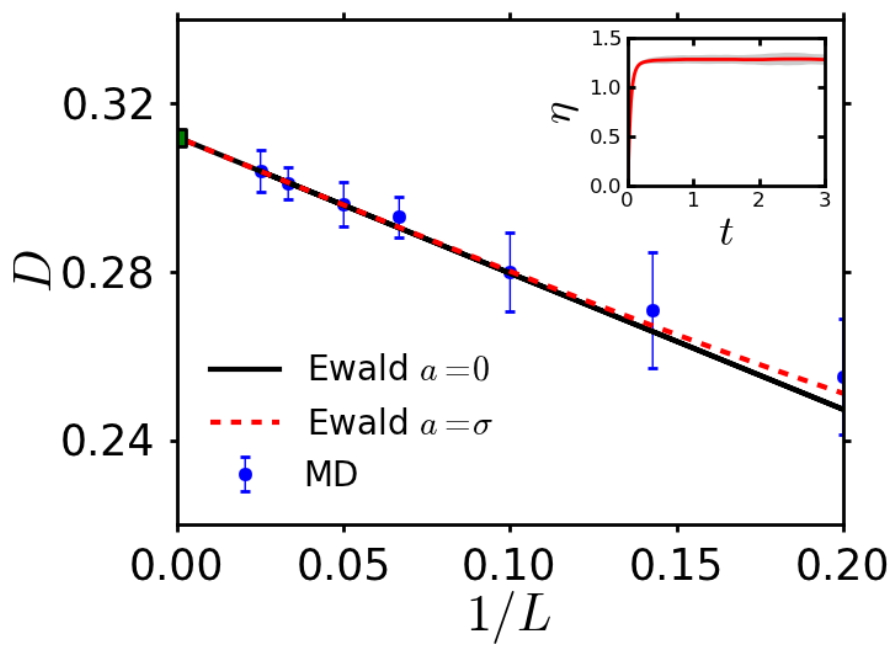

Figure 2. Diffusion coefficient for isotropic systems $\left(L_{\|}=L_{\perp}=L\right)$. Simulation results (blue symbols) are compared to the hydrodynamic calculations for a point particle (Eq. 1, black solid line) and with a size equal to the LJ diameter ( $a=\sigma$ in Eqs. 5-7, red dashed line). The inset shows the integral defining the viscosity in the Green-Kubo relation Eq. 10 (with errorbars in light grey). The corresponding value of the viscosity is used for the hydrodynamic calculations and the only fitted parameter is the extrapolation to infinite box size $D_{\infty}$ (indicated by a green square), used in the following. All quantities are given in LJ units.

For box sizes comparable to the LJ diameter, one observes deviations from Eq. 1, as expected for this limit of point particles. Nevertheless the effect of the finite particle size is moderate, as can also been seen in Figure 2 which reports the exact numerical result for a size $a=\sigma$ (the relative correction is of order $a^{2} / L^{2}$, as shown in Ref. [1]). For $L \geq 10 \sigma$, this effect can be neglected, as we will do in the following. 


\section{Anisotropic boxes}

We now turn to the more general case of anisotropic boxes and consider first the evolution of the components of the diffusion tensor $D_{\|}$and $D_{\perp}$ for fixed values of $L_{\|}$, as a function of the perpendicular dimension $L_{\perp}$. Figure 3 reports results for $L_{\|}=10$ and $40 \sigma$. One first observes that the $x$ and $y$ components are equal, and that $D_{\|}$and $D_{\perp}$ differ, as expected from the symmetry of the simulation cell. In addition, none of these components follow the simple scaling (Eq. 1) of the isotropic case. $D_{\|}$and $D_{\perp}$ may decrease or increase, simultaneously or in opposite directions, depending on the size and aspect ratio. Both curves intersect as expected when $L_{\perp}=L_{\|}$. A striking observation is that both components may exceed the value $D_{\infty}$ for an infinite cubic cell, whereas hydrodynamic effects can only reduce the diffusion coefficient for isotropic case.
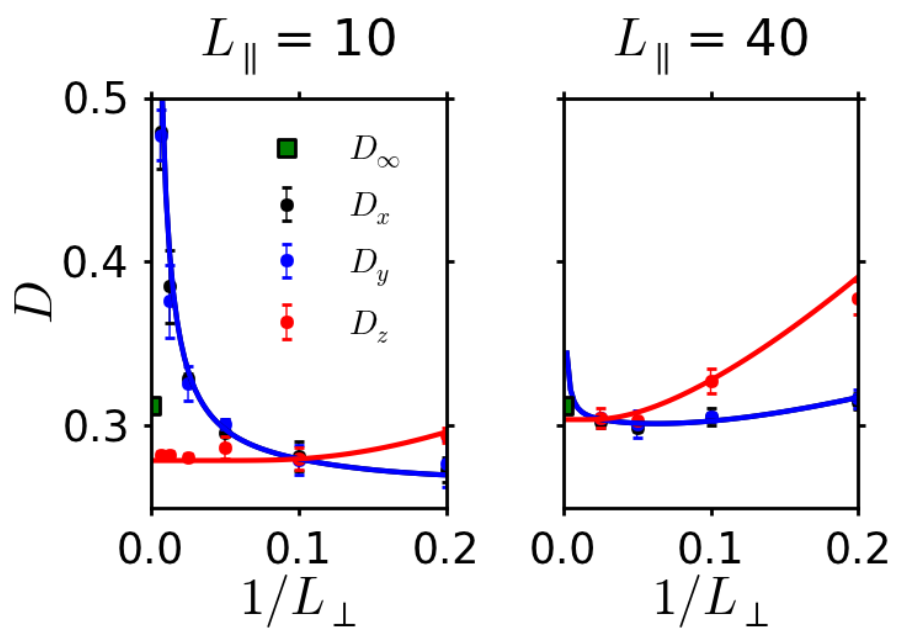

Figure 3. Diagonal components of the diffusion tensor, for $L_{x}=L_{y}=L_{\|}=10$ and 40 , as a function of $1 / L_{z}=1 / L_{\perp}$. Simulation results (symbols) are compared to the hydrodynamic result for a point particle ( $a=0$ in Eqs. $5-7$, lines). The diffusion coefficient extrapolated for an infinite isotropic box size, $D_{\infty}$, is also indicated (green square). All quantities are in LJ units.

Finally, we note that the hydrodynamic calculations for point particles $(a=0$ in Eqs. 5-7) perfectly reproduce the simulation results. This indicates that although the anisotropic case is more complex than the isotropic one, all the finite size effects are due to hydrodynamics, at least in this regime where $L_{\|}$and $L_{\perp}$ are larger than $5 \sigma$. All the above discussion also applies to the evolution of $D_{\|}$and $D_{\perp}$ for fixed values of $L_{\perp}$, as a function of the perpendicular dimension $L_{\|}$, as can be seen for $L_{\|}=10$ and 40 in Figure 3.

\subsection{Scaling with size and aspect ratio}

It may seem surprising that some components exceed in some cases the value for an infinite cubic cell and even seem to diverge e.g. as $L_{\perp} \rightarrow \infty$ for $L_{\|}=10 \sigma$ (Figure 3). One might expect, for example, to recover $D_{\infty}$ for an infinitely large system. For example, comparing the $L_{\perp} \rightarrow \infty$ (resp. $L_{\|} \rightarrow \infty$ ) for $L_{\|}=40 \sigma$ (resp. $L_{\perp}$ ) to the same limit for $10 \sigma$, one already sees that both $D_{\perp}$ and $D_{\|}$approach $D_{\infty}$. However, for an anisotropic system the proper macroscopic limit should be taken at fixed aspect ratio $\alpha=L_{\perp} / L_{\|}$. Examination of Eqs. 5-7 shows that both 

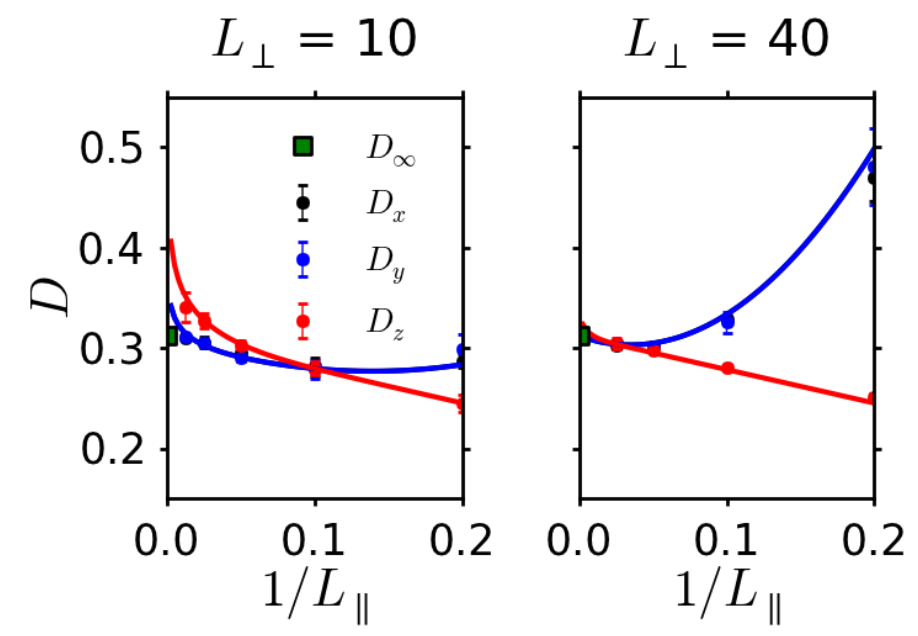

Figure 4. Diagonal components of the diffusion tensor, for $L_{z}=L_{\perp}=10$ and 40 , as a function of $1 / L_{x}=1 / L_{y}=1 / L_{\|}$. Simulation results (symbols) are compared to the hydrodynamic result for a point particle ( $a=0$ in Eqs. 5-7, lines). The diffusion coefficient extrapolated for an infinite isotropic box size, $D_{\infty}$, is also indicated (green square). All quantities are in LJ units.

component of the diffusion tensor can be rewritten as:

$$
D_{\|, \perp}=D_{\infty}+\frac{k_{B} T}{6 \pi \eta L_{\|}} g_{\|, \perp}\left(\xi L_{\|}, \frac{L_{\perp}}{L_{\|}}, \frac{a}{L_{\|}}\right)
$$

where the two functions $g_{\|, \perp}$ can be deduced straitforwardly from the above expressions. Noting that the Ewald summation technique is independent of the choice of $\xi$ (when both sums extend over the complete real and reciprocal space), these two functions are in fact independent of $\xi L_{\|}$. In addition, we have seen in the previous section that molecular simulation results are perfectly reproduced, for the cases considered here $\left(a / L_{\|} \sim \sigma / L_{\|}<1 / 5\right)$, by the hydrodynamic result for point particles $(a=0)$. In this limit, we thus obtain the following scaling:

$$
D_{\|, \perp}=D_{\infty}+\frac{k_{B} T}{6 \pi \eta L_{\|}} h_{\|, \perp}\left(\frac{L_{\perp}}{L_{\|}}\right)
$$

where the two functions $h_{\|, \perp}$ now depend only on the aspect ratio $\alpha=L_{\perp} / L_{\|}$. Both functions can be determined numerically and the isotropic case already provides $h_{\|, \perp}(1)=-c \approx-2.837$.

Figure 5 reports the scaling functions $h_{\|, \perp}=\left(D_{\|, \perp}-D_{\infty}\right) /\left(k_{B} T / 6 \pi \eta L_{\|}\right)$as a function of the aspect ratio for all simulated systems. They all collapse on a master curve (one for each component), which is perfectly described by the hydrodynamic result of Eqs. 5-7.

This figure not only confirms the hydrodynamic origin of the non-trivial finitesize effect on the diffusion tensor in anisotropic simulation cells, but also provides the evolution of the scaling functions $h_{\|, \perp}$. For the perpendicular component, $h_{\perp}$ (red curve in Figure 5) decreases monotonously and plateaus for large aspect ratios (thin rod), with $\lim _{\alpha \rightarrow \infty} h_{\perp} \approx-2.925$, compared to $c \approx-2.873$ for $\alpha=1$ (cubic). For small aspect ratios (flat cell), $h_{\perp}$ diverges approximately as $\alpha^{-2}$. The finitesize correction is positive $\left(D_{\perp}>D_{\infty}\right)$ for $\alpha<\alpha_{\perp}^{0} \approx 0.396$, while it is negative otherwise. As for the parallel component, $h_{\|}$(blue curve in Figure 5) decreases for 


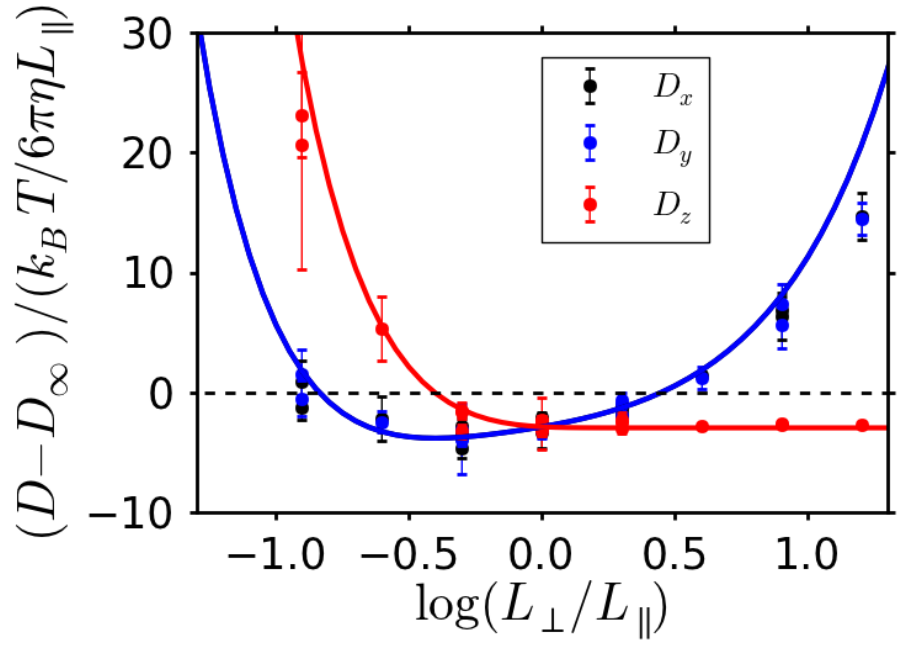

Figure 5. Scaling functions $h_{\|, \perp}=\left(D_{\|, \perp}-D_{\infty}\right) /\left(k_{B} T / 6 \pi \eta L_{\|}\right)$as a function of the aspect ratio $\alpha=L_{\perp} / L_{\|}$. Simulation results for all systems (see Table 1) are reported (symbols) together with the hydrodynamic result Eqs. 5-7. Note the logarithmic scale on the $x$-axis.

$\alpha<\alpha_{\|}^{m} \approx 0.396$ down to $h_{\|}\left(\alpha_{\|}^{m}\right) \approx-3.788$ and increases for $\alpha>\alpha_{\|}^{m}$. It diverges approximately as $\alpha^{-2}$ for $\alpha \rightarrow 0$ and as $\alpha$ for $\alpha \rightarrow \infty$. Finally, the finite-size correction is positive $\left(D_{\|}>D_{\infty}\right)$ for $\alpha<\alpha_{\|}^{1} \approx 0.146$ or $\alpha>\alpha_{\|}^{2} \approx 2.794$ and negative otherwise. The fact that the minimum of $h_{\|}$is obtained for a value $\alpha_{\|}^{m}$ approximately equal to the zero $\alpha_{\perp}^{0}$ of $h_{\perp}$ is striking, but we were not able to demonstrate the equality between these two particular points.

\section{Conclusion}

We have investigated systematically the effect of the cell size and shape on the diffusion properties in MD simulations. Specifically, we considered a bulk LJ fluid in orthorhombic cells with one length differing from the other two. We showed that the components of the diffusion tensor display complex variations as a function of the two independent lengths and may even become in some cases larger than the macroscopic limit for a cubic cell (for which the finite size effect is always a decrease in the diffusion coefficient). These variations are perfectly described by a purely hydrodynamic theory, which extends results obtained previously for the anisotropic case. In particular, we provided the explicit expression of the diffusion tensor including the effect of the finite size of the diffusing particle. The latter turns out to be small in the present case, but may be more important for solutes larger than solvent molecules in small simulation cells. Using this hydrodynamic description, we have further shown that the simulation results follow a simple scaling as a function of box size and aspect ratio and the corresponding scaling functions have been determined numerically. These findings should have implications for confined fluids (see e.g. Ref. [17]): In that case hydrodynamic flows in the directions parallel to the confining walls may result in spurious size effects on the computed diffusion coefficients. The approach followed in the present work should allow to adress this more practically important case. 


\section{Acknowledgements}

The authors would like express their gratitude to Jean-Pierre Hansen for his guidance and their pleasure to enjoy him as a colleague at UPMC. BR is particularly indebted to Jean-Pierre for his continuing scientific inspiration and friendship, from his first interactions with him as a visiting student in Cambridge to a shared office in Paris.

\section{References}

[1] I.C. Yeh and G. Hummer, The Journal of Physical Chemistry B 108 (40), 15873 (2004).

[2] B. Dünweg and K. Kremer, The Journal of chemical physics 99 (9), 6983 (1993).

[3] J. Rotne and S. Prager, The Journal of Chemical Physics 50 (11), 4831 (1969).

[4] H. Hasimoto, Journal of Fluid Mechanics 5 (2), 317 (1959).

[5] S. Tazi, A. Boţan, M. Salanne, V. Marry, P. Turq and B. Rotenberg, Journal of Physics: Condensed Matter 24 (28), 284117 (2012).

[6] D. Rozmanov and P.G. Kusalik, The Journal of Chemical Physics 136 (4), 044507 (2012).

[7] L.P. Wang, T. Head-Gordon, J.W. Ponder, P. Ren, J.D. Chodera, P.K. Eastman, T.J. Martinez and V.S. Pande, The Journal of Physical Chemistry B 117 (34), 9956 (2013).

[8] S. Gabl, C. Schröder and O. Steinhauser, The Journal of Chemical Physics 137 (9), 094501 (2012).

[9] S.P. Singh, C.C. Huang, E. Westphal, G. Gompper and R.G. Winkler, The Journal of Chemical Physics 141 (8), 084901 (2014).

[10] B. Charbonneau, P. Charbonneau, Y. Jin, G. Parisi and F. Zamponi, The Journal of Chemical Physics 139 (16), 164502 (2013).

[11] S. Kerisit and C. Liu, Geochimica et Cosmochimica Acta 74 (17), 4937 (2010).

[12] S. Tazi, J.J. Molina, B. Rotenberg, P. Turq, R. Vuilleumier and M. Salanne, The Journal of Chemical Physics 136 (11), 114507 (2012).

[13] T.D. Kühne, M. Krack and M. Parrinello, Journal of Chemical Theory and Computation 5 (2), 235 (2009).

[14] C.W.J. Beenakker, The Journal of chemical physics 85 (3), 1581 (1986).

[15] H. Flyvbjerg and H.G. Petersen, The Journal of Chemical Physics 91 (1), 461 (1989).

[16] LAMMPS, http://lammps.sandia.gov.

[17] M. Holmboe and I.C. Bourg, The Journal of Physical Chemistry C 118 (2), 1001 (2014). 